\title{
SMOOTHNESS CONDITIONS AND INTEGRABILITY THEOREMS ON BOUNDED VILENKIN GROUPS
}

\author{
WALTER R. BLOOM and JOHN J. F. FOURNIER
}

(Received 6 March 1987)

Communicated by J. F. Price Dedicated to Robert Edwards in recognition of
25 years' distinguished contribution to mathematics in Australia,
on the occasion of his retirement

\begin{abstract}
Various criteria, in terms of forward differences and related operations on coefficients, are shown to imply that certain series on bounded Vilenkin groups represent integrable functions. These results include analogues of known integrability theorems for trigonometric series. The method of proof is to pass from the given series to a derived series, and to deduce the integrability of the original series from smoothness properties of the latter.
\end{abstract}

1980 Mathematics subject classification (Amer. Math. Soc.): primary 42 C 10; secondary 43 A 50,43 A 70.

\section{Conditions on coefficients}

We will specify our conventions concerning Vilenkin systems and Vilenkin groups in Sections 2 and 3. The main fact that we need in this section is that there is a measure-preserving correspondence between such groups and the interval $[0,1)$. This allows us to state our main theorem for series on $[0,1)$ in this section, and to prove the theorems in Section 4 by proving the corresponding statement about

Research partly carried out while the first author held an Alexander von Humboldt fellowship at the University of Tübingen.

Research of the second author partially supported by N.S.E.R.C. operating grant number 4822 . (C) 1988 Australian Mathematical Society 0263-6115/88 \$A2.00 + 0.00 
series on bounded Vilenkin groups. In the proof we will use certain facts about smoothness classes on Vilenkin groups, which we summarize in Section 3.

The symbol $\left(\chi_{n}\right)_{n=0}^{\infty}$ will denote an orthogonal sequence of functions on the interval $[0,1)$ with the property that $\sup _{n}\left\|\chi_{n}\right\|_{\infty}<\infty$. Given such a sequence $\left(\chi_{n}\right)$ and an integrable function $f$ on $[0,1)$ let

$$
\hat{f}(n)=\int_{0}^{1} f(t) \bar{\chi}_{n}(t) d t .
$$

Given a sequence $\left(a_{n}\right)_{n=0}^{\infty}$ of scalars we say that the series $\sum_{n=0}^{\infty} a_{n} \chi_{n}$ represents an integrable function if there is a function $f$ in $L^{1}[0,1)$ such that $\hat{f}(n)=a_{n}$ for all $n$. By the Riemann-Lebesgue lemma a necessary condition for this is that $a_{n} \rightarrow 0$ as $n \rightarrow \infty$, that is, that the coefficient sequence $\left(a_{n}\right)$ belongs to the space $c_{0}(N)$. This condition is not sufficient, however, and we seek further conditions on the coefficients $\left(a_{n}\right)$ that guarantee that the series $\sum_{n=0}^{\infty} a_{n} \chi_{n}$ represents an integrable function.

For each nonnegative integer $n$ let $\Delta a_{n}=a_{n+1}-a_{n}$. Fix a sequence $\left(m_{r}\right)_{r=0}^{\infty}$ of positive integers with the properties that $m_{0}=1$,

$$
\limsup _{r \rightarrow \infty} \frac{m_{r+1}}{m_{r}}<\infty \text { and } \quad \liminf _{r \rightarrow \infty} \frac{m_{r+1}}{m_{r}}>1 \text {. }
$$

Then, given an index $p$ in the interval $[1, \infty)$, say that the sequence $\left(a_{n}\right)$ satisfies the condition $\mathcal{F}_{p}$ if

$$
\sum_{r=1}^{\infty} m_{r}\left\{\frac{1}{m_{r}} \sum_{m_{r-1} \leq n<m_{r}}\left|\Delta a_{n}\right|^{p}\right\}^{1 / p}<\infty .
$$

Similarly say that $\left(a_{n}\right)$ satisfies the condition $\mathcal{F}_{\infty}$ if

$$
\sum_{r=1}^{\infty} m_{r} \sup _{m_{r-1} \leq n<m_{r}}\left|\Delta a_{n}\right|<\infty .
$$

Finally say that $\left(a_{n}\right)$ satisfies condition $\mathcal{F}$ if $a_{n} \rightarrow 0$ as $n \rightarrow \infty$ and there is an index $p>1$ for which $\left(a_{n}\right)$ satisfies condition $\mathcal{F}_{p}$.

In Section 2 we will describe a class of orthonormal uniformly bounded sequences of functions on the interval $[0,1)$, called bounded Vilenkin systems. We will prove the following assertion in Section 4.

THEOREM 1. If $\left(\chi_{n}\right)$ is a bounded Vilenkin system and if $\left(a_{n}\right)$ is a sequence of scalars satisfying condition $₹$ then the series $\sum_{n=0}^{\infty} a_{n} \chi_{n}$ represents an integrable function.

We conclude this section with some remarks about Theorem 1 . The bestknown example of a Vilenkin system is the Walsh system. Bruce Aubertin has 
found a different proof of the theorem in this special case; we thank him for showing us his proof and for some very useful comments about the problem. The model for our theorem is the result of G. A. Fomin [6] to the effect that if $\left(a_{n}\right)$ satisfies condition $\mathcal{F}$ then the series $\sum_{n=0}^{\infty} a_{n} \cos (2 \pi n t)$ represents an integrable function. Fomin also showed for the same condition on $\left(a_{n}\right)$ that the corresponding sine series represents an integrable function if and only if $\sum_{n=1}^{\infty}\left(\left|a_{n}\right| / n\right)<\infty$. We will encounter an analogue of the latter condition in our proof of Theorem 1, but it will turn out for bounded Vilenkin systems that the analogue is automatically satisfied. Other proofs of Fomin's theorems have appeared in [4] and [7].

Observe that if a coefficient sequence $\left(a_{n}\right)$ satisfies condition $\mathcal{F}_{p}$ for one choice of the sequence $\left(m_{r}\right)$ then $\left(a_{n}\right)$ also satisfies condition $\mathcal{F}_{p}$ for all other choices of $\left(m_{r}\right)$ with the properties in (1). It follows easily from Hölder's inequality that if $\left(a_{n}\right)$ satisfies condition $\mathcal{F}_{p}$ for an index $p>1$ then it also satisfies condition $\mathcal{F}_{q}$ for all $q<p$. In particular $\mathcal{F}_{\infty}$ is the strongest of these conditions and $\mathcal{F}_{1}$ is the weakest. The latter condition can be written more simply in the form $\sum_{n=1}^{\infty}\left|\Delta a_{n}\right|<\infty$; in other words the sequence $\left(a_{n}\right)$ satisfies condition $\mathcal{F}_{1}$ if and only if it is of bounded variation.

It is a classical fact [5, Section 7.3.1] that if the sequence $\left(a_{n}\right)$ is convex, that is, if $\Delta^{2} a_{n} \geq 0$ for all $n$, and if $a_{n} \rightarrow 0$ as $n \rightarrow \infty$, then the series $\sum_{n=0}^{\infty} a_{n} \cos (2 \pi n t)$ represents an integrable function. This is a special case of Fomin's theorem because every convex sequence in the space $c_{0}(N)$ satisfies condition $F_{\infty}$. Condition $₹$ also includes several other classical sufficient conditions for integrability of cosine series [4]. Our Theorem 1 implies that these conditions also suffice for integrability of Walsh series. It was already known [10] that convexity is sufficient, as is a weaker condition called quasiconvexity, which is also a special case of condition $\mathcal{F}$. Finally it should be observed that condition $\mathcal{F}$ in Theorem 1 cannot be weakened to include the endpoint case $p=1$; indeed in [1] examples are given of sequences $\left(a_{n}\right)$ having bounded variation, belonging to the space $c_{0}(N)$, and for which the Walsh series with coefficients $\left(a_{n}\right)$ does not represent an integrable function.

\section{Vilenkin systems}

In this section we will set out our notation for Vilenkin systems and discuss some properties of these. We refer to [9] for more on this topic. We will use the term Vilenkin system for any orthonormal system of functions that can be built up in the following way.

Begin with a nonatomic probability space and a sequence $\left(p_{n}\right)_{n=0}^{\infty}$ of prime numbers, and construct a sequence $\left(\chi_{n}\right)$ of functions on the space as follows. Let 
$\chi_{0}$ be the constant function 1 and let $\Gamma_{0}$ be the singleton set $\left\{\chi_{0}\right\}$. Let $\phi_{1}$ be a function taking each value in the set of $p_{1}$ th roots of unity with probability $1 / p_{1}$. For $1 \leq n<p_{1}$ let $\chi_{n}$ be the function $\phi_{1}^{n}$, and let $\Gamma_{1}$ be the set of all functions $\chi_{n}$ with $n<p_{1}$. For each positive integer $r$ let $m_{r}=\prod_{n=1}^{r} p_{n}$ and let $m_{0}=1$. Assume that the functions $\chi_{n}$ with $n<m_{r-1}$ have been specified and denote the set of these by $\Gamma_{r-1}$. Then select three functions $\alpha_{r}, \beta_{r}$ and $\gamma_{r}$ with the following properties:

(i) $\alpha_{r}$ belongs to the set $\Gamma_{r-1}$;

(ii) $\beta_{r}$ is a $p_{r}$ th root of $\alpha_{r}$ that is constant on each set where $\alpha_{r}$ is constant;

(iii) $\gamma_{r}$ takes each value in the set of $p_{r}$ th roots of unity with probability $1 / p_{r}$;

(iv) $\gamma_{r}$ is independent of the functions in the set $\Gamma_{r-1}$.

Let $\phi_{r}$ be the product $\gamma_{r} \beta_{r}$. Then $\phi_{r}^{p_{r}}$ belongs to the set $\Gamma_{r-1}$. The values taken by $\phi_{r}$ form a subgroup of the circle group, each occurring with the same probability. Observe that each integer $n$ in the interval $\left[m_{r-1}, m_{r}\right)$ has a unique representation as $n=j m_{r-1}+l$ with $1 \leq j<p_{r}$ and $0 \leq l<m_{r-1}$. Given this representation of $n$ let $\chi_{n}=\phi_{r}{ }^{j} \chi_{l}$. Denote by $\Gamma_{r}$ the set of functions $\chi_{n}$ with $n<m_{r}$ and continue the construction for each $r$. Call the system of functions constructed in this way bounded if the sequence $\left(p_{n}\right)$ is bounded.

The simplest example of such a system of functions is the Walsh system, which arises when $p_{n}=2$ for all $n$ and each of the functions $\phi_{n}$ takes the values \pm 1 only, each with probability $1 / 2$. The most common representation of this system is as a set of functions on the interval $[0,1)$, and it is usually specified that the function $\phi_{1}$ is equal to 1 on the first half of this interval and to -1 on the second half, and that $\phi_{2}$ is equal to 1 on the first halves of each of the intervals $[0,1 / 2)$ and $[1 / 2,1)$ and to -1 on the second halves of these intervals etc. In this example $\phi_{r}^{2}=1$ for all $r$; other examples of Vilenkin systems, still with $p_{n}=2$ for all $n$, arise by letting $\phi_{r}^{2}$ coincide with some other function in the set $\Gamma_{r-1}$. Varying the sequence $\left(p_{n}\right)$ leads to further examples.

What determines the integrability of a given Vilenkin series is not the particular representation that we choose for the underlying probability space, but rather the joint distribution of the functions $\chi_{n}$ and the properties of the coefficients in the series. In proving that certain conditions on the coefficients are sufficient for integrability we will find it convenient to view the underlying probability space as a totally disconnected abelian group. This is the point of view that we will take in the next two sections. In this section, however, we concentrate on the properties of Vilenkin systems that are independent of the representation of the underlying probability space. We may assume with no real loss of generality that this space is the interval $[0,1)$ with normalized Lebesgue measure.

The function $D_{N}=\sum_{n=0}^{N-1} \chi_{n}$ is called the Dirichlet kernel of order $N$. When $N=m_{r}$ for some integer $r$ this kernel has a particularly simple form. Let $G_{r}$ be the set where the functions $\chi_{n}$ with $n<m_{r}$ all coincide with 1 . It is evident 
that $D_{m_{r}}=m_{r}$ on the set $G_{r}$, and it turns out that $D_{m_{r}}$ vanishes elsewhere; that is, letting $\xi_{G_{r}}$ denote the indicator function of the set $G_{r}$ we have that $D_{m_{r}}=m_{r} \xi_{G_{r}}$. Similarly any sum of the form $\sum_{j m_{r} \leq n<(j+1) m_{r}} \chi_{n}$ vanishes off the set $G_{r}$ and coincides on that set with $m_{r} \chi_{n}$ for any one of the functions $\chi_{n}$ appearing in the sum.

The set $G_{r}$ has measure $1 / m_{r}$. When we represent the underlying space as the interval $[0,1)$ we can arrange matters so that $G_{r}=\left[0,1 / m_{r}\right)$ for all $r$. In this setting our proof of Theorem 1 runs roughly as follows. We choose a suitable function $k$ such that $|k(t)| \geq t$ for all $t$ in $[0,1)$ and such that the formal product of the given series $\sum_{n=0}^{\infty} a_{n} \chi_{n}$ and the Fourier series for $k$, with respect to the given Vilenkin system, is well-defined. We show that this product is the Fourier series of a function $f$ with the property that

$$
\int_{0}^{1} \frac{|f(t)|}{t} d t<\infty
$$

It follows that the ratio $f / k$ belongs to $L^{1}[0,1)$, and we then show that the given series $\sum_{n=0}^{\infty} a_{n} \chi_{n}$ is just the Fourier series of this integrable function.

The roundabout approach to the integrability of the Vilenkin series is motivated by the fact that a similar procedure works well for cosine series [7]. Our way of showing that inequality (2) holds is to arrange for the function $f$ to vanish at 0 and to be smooth enough that the inequality follows. The notion of smoothness that we use is most easily described in the setting of Vilenkin groups, and this we present in the next section.

\section{Smoothness classes on Vilenkin groups}

We now summarize the facts that we need concerning Vilenkin groups and certain smoothness classes on them. For a particular Vilenkin system denote the set of all functions $\chi_{n}$ by $\Gamma$. One of the key properties of such a system is that the product of any two functions in the set $\Gamma$ also belongs to $\Gamma$, as does the reciprocal of any function in $\Gamma$. Thus the set $\Gamma$ is a group under pointwise multiplication; moreover each of the sets $\Gamma_{r}$ is a subgroup of $\Gamma$. Put the discrete topology on the abelian group $\Gamma$. Then $\Gamma$ has a compact abelian dual group, $G$ say and, since the dual group of $G$ is isomorphic to $\Gamma$, each element of $\Gamma$ can be regarded as a function on $G$. The Haar measure on $G$ can be normalized so that $G$ has total mass 1; this provides a representation of $\Gamma$ as a set of functions on a probability space that is also a compact abelian group.

The advantage of this representation is that it provides a notion of translation of functions that is consistent with the multiplicative structure on $\Gamma$. Write the group operation on $G$ additively and denote the identity element of $G$ by 
0. Given a function $f$ on $G$ and $y \in G$, let $\tau_{y} f$ be the function for which $\tau_{y} f(x)=f(x-y)$ for all $x \in G$. If $f \in L^{p}(G)$ for some index $p$ in the interval $[1, \infty)$ then $\left\|\tau_{y} f-f\right\|_{p} \rightarrow 0$ as $y \rightarrow 0$, and the rate at which $\left\|\tau_{y} f-f\right\|_{p}$ tends to 0 is a measure of the smoothness of the function $f$.

Again let $G_{r}=\left\{x \in G: \chi_{n}(x)=1\right.$ for all $\left.n<m_{r}\right\}$. Now $G_{r}$ is a subgroup of $G$, called the annihilator of $\Gamma_{r}$. The discrete group $\Gamma$ is the union of the increasing sequence $\left(\Gamma_{r}\right)$; dually the groups $G_{r}$ form a decreasing sequence of subgroups of $G$ with intersection $\{0\}$. Each of the subgroups $G_{r}$ is open and closed in the topology on $G$ and the set of all translates of these subgroups forms a basis for that topology. The index of $G_{r}$ in $G_{r-1}$ is the same as the index of $\Gamma_{r-1}$ in $\Gamma_{r}$, that is, the prime number $p_{r}$. The group $G$ is called a Vilenkin group, and it is called bounded if the sequence $\left(p_{r}\right)$ is bounded. The topology on $G$ can also be specified by a metric, for example by letting $d(x, y)=1 / m_{r+1}$ whenever $x-y$ belongs to $G_{r}$ but not to $G_{r+1}$.

Conversely one can start with any separable infinite totally disconnected compact abelian group $G$ and show that there is a nested sequence $\left(G_{r}\right)$ of open subgroups of $G$ that intersect in the set $\{0\}$, whose translates form a basis for the topology on $G$, and for which $G_{r}$ has prime index in $G_{r-1}$ for all $r$. The dual group of $G$ is then a Vilenkin system as defined in the previous section.

Given a number $p$ in the interval $[1, \infty)$ the $L^{p}$-modulus of continuity of a function $f$ in $L^{p}(G)$ is defined by letting $\omega_{p}(f, t)=\sup \left\{\left\|\tau_{y} f-f\right\|_{p}: d(y, 0) \leq t\right\}$ for all $t \in[0,1)$. Given $\alpha \in(0,1]$ and $q \in[1, \infty)$ let

$$
\|f\|_{\alpha, p, q}=\|f\|_{p}+\left\{\int_{0}^{1}\left(t^{-\alpha} \omega_{p}(f, t)\right)^{q} \frac{d t}{t}\right\}^{1 / q} .
$$

Define the generalized Lipschitz space $\Lambda(\alpha, p, q)$ to be the set of all functions $f$ in $L^{p}(G)$ for which the quantity $\|f\|_{\alpha, p, q}$ is finite.

These spaces have been studied for some time, and it is known [3] for $p<\infty$ that if $f \in \Lambda(1 / p, p, 1)$ then $f$ coincides almost everywhere with a continuous function, and for fixed $p$ the space $\Lambda(1 / p, p, 1)$ is the largest of the space $\Lambda(\alpha, p, q)$ with this property. Moreover when $1 \leq p \leq 2$ every function in $\Lambda(1 / p, p, 1)$ has an absolutely convergent Fourier series, and when $2<p<\infty$ and the group is bounded every function in $\Lambda(1 / p, p, 1)$ has a uniformly convergent Fourier series [3].

It is shown in [3] that there is a simple criterion on the Fourier coefficients of a function $f$ in $\Lambda(1 / p, p, 1)$ that determines whether the function $x \rightarrow f(x) / d(x, 0)$ is integrable. Denote the partial sums of the Fourier series of $f$ by $S_{N} f=$ $\sum_{n=0}^{N-1} \hat{f}\left(\chi_{n}\right) \chi_{n}$.

THEOREM 2. Suppose that $f$ is a function on a bounded Vilenkin group and that $f \in \Lambda(1 / p, p, 1)$ for some $p \in[1, \infty)$. Then the following conditions are 
equivalent

$$
\begin{aligned}
& \int_{G} \frac{|f(x)|}{d(x, 0)} d x<\infty . \\
& \sum_{N=1}^{\infty} \frac{\left|S_{N} f(0)\right|}{N}<\infty \\
& \sum_{r=0}^{\infty}\left|S_{m_{r}} f(0)\right|<\infty .
\end{aligned}
$$

In using this theorem it is useful to have a criterion, in terms of its Fourier coefficients, for an integrable function $f$ to belong to the space $\Lambda(1 / p, p, 1)$. To this end we vary the indexing in [3] slightly and let

$$
f_{r}=S_{m_{r}} f-S_{m_{r-1}} f=\sum_{m_{r-1} \leq n<m_{r}} \hat{f}\left(\chi_{n}\right) \chi_{n} .
$$

When $0<\alpha<1$ and $1 \leq p, q<\infty$ define the Besov space $B(\alpha, p, q)$ to be the set of all functions $f$ in $L^{p}(G)$ for which

$$
\sum_{r=0}^{\infty}\left[m_{r}{ }^{\alpha}\left\|f_{r}\right\|_{p}\right]^{1 / q}<\infty
$$

It is shown in [3] that $B(\alpha, p, q)=\Lambda(\alpha, p, q)$ for these values of the indices. Note that when $p=2$ the quantities $\left\|f_{r}\right\|_{2}$ appearing above can be replaced by the $l^{2}$-norms of the Fourier coefficients of these functions.

At one point in the next section we will need the fact that conditions (i) and (iii) in Theorem 2 are equivalent for some functions that do not satisfy the hypothesis of the theorem. Let $B(0, \infty, 1)$ be the space of all continuous functions $f$ on $G$ with the property that $\sum_{r=1}^{\infty}\left\|f_{r}\right\|_{\infty}<\infty$. It is pointed out in [3] that for functions $f$ in this space on a bounded Vilenkin group conditions (i) and (iii) are equivalent provided that

$$
\sum_{r=1}^{\infty} \int_{G \backslash G_{r}}\left|f_{r}(x)\right| \frac{d x}{d(x, 0)}<\infty
$$

\section{Integrability theorems}

We need the notion of formal multiplication of Vilenkin series. In this context it is best to regard the coefficients in the series as functions on the set $\Gamma$ rather than on the set $\{0,1,2, \ldots\}$ that indexes $\Gamma$. Thus we write $a\left(\chi_{n}\right)$ in place of $a_{n}$. 
Recall that the convolution of two functions $a$ and $b$ on the group $\Gamma$ is defined by

$$
c\left(\chi_{n}\right)=a * b\left(\chi_{n}\right)=\sum_{m=0}^{\infty} a\left(\chi_{n} \bar{\chi}_{m}\right) b\left(\chi_{m}\right)
$$

whenever the series on the right converges. The formal product of two Vilenkin series with coefficients $a$ and $b$ is the series with coefficients $a * b$. In particular this product is defined when $a \in l^{\infty}(\Gamma)$ and $b \in l^{1}(\Gamma)$.

Denote by $A(G)$ the algebra of all functions $f$ on $G$ for which $\hat{f} \in l^{1}(\Gamma)$. Call a function $k$ on $G$ adapted if $k \in A(G)$ and there are positive constants $\kappa$ and $K$ such that

$$
\kappa d(x, 0) \leq|k(x)| \leq K d(x, 0)
$$

for all $x$ in $G$. We will give an example of such a function later in this section.

THEOREM 3. Let $\left(\chi_{n}\right)$ be a bounded Vilenkin system and let $\left(a_{n}\right) \in c_{0}(\Gamma)$. Let $k$ be an adapted function. Suppose that the formal product of the Fourier series of $k$ and the series $\sum_{n=0}^{\infty} a_{n} \chi_{n}$ is the Fourier series of a function $f$ that belongs to one of the spaces $\Lambda(1 / p, p, 1)$ with $p<\infty$. Then the series $\sum_{n=0}^{\infty} a_{n} \chi_{n}$ represents an integrable function if and only if

$$
\sum_{r=0}^{\infty}\left|S_{m_{r}} f(0)\right|<\infty
$$

ProoF. If the series $\sum_{n=0}^{\infty} a_{n} \chi_{n}$ represents an integrable function, $F$ say, then the function $f$ really is the product $k F$. Since $k$ is adapted and $F$ is integrable

$$
\int_{G} \frac{|f(x)|}{d(x, 0)} d x<\infty
$$

By Theorem 2 this condition is equivalent to inequality (5).

On the other hand if inequality (5) holds then so does inequality (6), and it follows that the function $f / k$ is integrable. The one point that remains to be verified is that the Fourier series of $f / k$ is $\sum_{n=0}^{\infty} a_{n} \chi_{n}$. Recall that a pseudofunction is simply a Vilenkin series with coefficients that belong to $c_{0}(\Gamma)$, and that a pseudomeasure is a Vilenkin series with bounded coefficients. Let $F$ be the pseudofunction $\sum_{n=0}^{\infty} a_{n} \chi_{n}$. The formal products of pseudomeasures with functions in $A(G)$ are always well-defined. Both $F$ and $f / k$ have the property that their product with $k$ is equal to $f$.

To deduce that $F=f / k$ we consider the support of a pseudomeasure. This is the complement in $G$ of the largest open set on which the pseudomeasure vanishes; a pseudomeasure, $\mu$ say, vanishes on an open set, $U$ say, if the formal product $g \cdot \mu$ is the trivial series for all functions $g$ in $A(G)$ that are supported 
by compact subsets of $U$. Let $\mu$ be the pseudofunction $F-f / k$. We claim that the support of $\mu$ is the singleton $\{0\}$ or is empty. If the claim is valid then $\mu$ must be a point mass at 0 and $\hat{\mu}$ must be constant. This constant must be 0 because $\hat{\mu} \in c_{0}(\Gamma)$, in which case $\mu=0$ and $F=f / k$, as required.

To prove the claim, let $C$ be a compact subset of the open set $G \backslash\{0\}$ and let $g$ be a function in $A(G)$ that vanishes off $C$. Since the function $k$ has no zeros in the set $C$ the Wiener-Lévy theorem yields a function $h$ in $A(G)$ with the property that $h k=1$ on $C$. Then $g=g h k$ and $g \cdot \mu=(g h)(k \cdot \mu)=(g h) 0=0$. Hence $\mu$ vanishes on the open set $G \backslash\{0\}$ as claimed. This completes the proof of the theorem.

We now give an example of an adapted function on a bounded Vilenkin group. For each integer $s \geq 2$ let $k_{s}$ be the product of the functions $\phi_{s}\left[1-\bar{\phi}_{s-1}\right]$ and $\left(1 / m_{s-2}\right)^{2} D_{m_{s-2}}$. Observe first that $\hat{k}_{s}\left(\chi_{n}\right)$ is equal to $\left(1 / m_{s-2}\right)^{2}$ for all $n$ in the interval $\left[m_{s-1}, m_{s-1}+m_{s-2}\right)$, to $-\left(1 / m_{s-2}\right)^{2}$ for all $n$ in $\left[2 m_{s-1}-m_{s-2}, 2 m_{s-1}\right)$, and to 0 for all other values of $n$. In particular $\left\|\hat{k}_{s}\right\|_{1}=2 / m_{s-2}$, so that the series $\sum_{s=2}^{\infty} k_{s}$ converges in the space $A(G)$ to some function $k$.

To verify that $k$ has property (4) for suitable constants $\kappa$ and $K$, note first that the function $k_{s}$ vanishes off $G_{s-2}$ because $D_{m_{s-2}}$ does, and that it vanishes on $G_{s-1}$ because $1-\bar{\phi}_{s-1}$ does. Moreover, $\phi_{s-1}^{p_{s-1}} \in \Gamma_{s-2}$ implies that $\phi_{s-1}^{p_{s-1}}=1$ on $G_{s-2}$. Therefore the values taken by the function $\phi_{s-1}$ on this set lie in the group of $p_{s-1}$ th roots of unity. On the other hand $G_{s-1}$ was defined as the set on which $\chi_{n}=1$ for all $n<m_{s-1}$, so that $\phi_{s-1} \neq 1$ on the corona $G_{s-2} \backslash G_{s-1}$. Hence the values taken by $\phi_{s-1}$ on this corona must be $p_{s-1}$ th roots of unity other than 1 and, in particular, $\left|1-\bar{\phi}_{s-1}\right|$ lies between $2 \sin \left(\pi / p_{s-1}\right)$ and 2 on this set. The other factor $\left(1 / m_{s-2}\right)^{2} D_{m_{s-2}}$ is equal to $1 / m_{s-2}$ on the same set. Since the sequence $\left(p_{s}\right)$ is bounded there are constants $\kappa$ and $K$ such that the function $k$ satisfies condition (4).

This adapted function was specifically devised for use in this paper but it turns out to be similar to one used by Onneweer [8] in a discussion of dyadic differentiation. Applying Theorem 3 with this choice of an adapted function yields an integrability criterion that has no counterpart for trigonometric series. It is also easy to prove the sufficiency of this condition via summation by parts.

THEOREM 4. Let $G$ be a bounded Vilenkin group and let a be a function in the space $c_{0}(\Gamma)$ that is constant on each of the sets $\Gamma_{r} \backslash \Gamma_{r-1}$ and satisfies condition $\mathcal{F}_{1}$. Then the series $\sum_{n=0}^{\infty} a\left(\chi_{n}\right) \chi_{n}$ represents an integrable function.

PROOF. For each nonnegative integer $r$ let $F_{r}=\sum_{m_{r-1} \leq n<m_{r}} a\left(\chi_{n}\right) \chi_{n}$ and consider the products of these functions with the various functions $k_{s}$. We claim that the function $F_{r}$ vanishes off the set $G_{r-1}$. To verify this we use the fact 
that $a$ is constant on the corona $\Gamma_{r} \backslash \Gamma_{r-1}$ to write $F_{r}$ in the form

$$
F_{r}=a\left(\chi_{m_{r-1}}\right) \sum_{j=1}^{p_{r}-1} \sum_{j m_{r-1} \leq n<(j+1) m_{r-1}} \chi_{n}
$$

As pointed out in Section 2 the inner sums above all vanish outside the set $G_{r-1}$. On the other hand the functions $k_{s}$ vanish on this set when $s \leq r$, so that the products $k_{s} F_{r}$ are all trivial for such values of $s$.

Next we make some observations that do not use any special properties of the coefficients $a\left(\chi_{n}\right)$. We adopt the convention that the functions $k_{s}$ are zero when $s \leq 1$ and we let $s \geq 2$ in the rest of this discussion. The transform of $F_{r}$ vanishes off the corona $\Gamma_{r} \backslash \Gamma_{r-1}$ and the transform of $k_{s}$ vanishes off the corona $\Gamma_{s} \backslash \Gamma_{s-1}$. Hence the transform of the product $k_{s} F_{r}$ vanishes off the product of these coronas. When $r<s$ this product set is included in the corona $\Gamma_{s} \backslash \Gamma_{s-1}$, and when $r>s$ it is included in $\Gamma_{r} \backslash \Gamma_{r-1}$; when $r=s$ the product set is included in the subgroup $\Gamma_{s}$.

Since $\hat{k}_{s}$ is constant on each coset of the subgroup $\Gamma_{s-2}$ the convolution $\hat{k}_{s} * b$ also has this property for all bounded functions $b$ on $\Gamma$. The value of $\hat{k}_{s} * b$ on such a coset $\chi_{j} \Gamma_{s-2}$ is

$$
\left(\frac{1}{m_{s-2}}\right)^{2}\left\{\sum_{\chi_{n} \in \bar{\phi} s \chi_{j} \Gamma_{s-2}} b\left(\chi_{n}\right)-\sum_{\chi_{n} \in \bar{\phi}_{s} \phi_{s-1} \chi_{j} \Gamma_{s-2}} b\left(\chi_{n}\right)\right\} .
$$

The fact that $k_{s} F_{r}=0$ when $s \leq r$ and $a$ is constant on $\Gamma_{r} \backslash \Gamma_{r-1}$ also follows from an analysis of the expression above with $b=a$ on $\Gamma_{r} \backslash \Gamma_{r-1}$ and $b=0$ elsewhere.

We now consider the products $k_{s} F_{r}$ when $s>r$. As noted above the transform of such a product vanishes outside the corona $\Gamma_{s} \backslash \Gamma_{s-1}$. In particular $S_{m_{t}}\left[k_{s} F_{r}\right](0)=0$ for all $t<s$. On the other hand the partial sum $S_{m_{s}}\left[k_{s} F_{r}\right](0)$ is equal to the sum of expressions of the form (7) as $j$ runs from 0 to $m_{s}-1$. As $j$ varies in this way each coset of $\Gamma_{s-2}$ that is included in $\Gamma_{s}$ occurs $m_{s-2}$ times as the support of the first sum in expression (7) and the same number of times as the support of the second sum in (7). Hence $S_{m_{s}}\left[k_{s} F_{r}\right](0)=0$. Finally the partial sums $S_{m_{t}}\left[k_{s} F_{r}\right](0)$ are also 0 when $t>s$ because the transform of $k_{s} F_{r}$ vanishes outside $\Gamma_{s}$.

Fix $s \geq 2$ and let $f_{s}=k_{s} \sum_{r=0}^{s-1} F_{r}$; then $S_{m_{t}} f_{s}(0)=0$ for all $t$. The Fourier coefficients of $f_{s}$ vanish outside $\Gamma_{s} \backslash \Gamma_{s-1}$. On this corona these coefficients are given by expression (7) in terms of differences of sums of values of the function $a$ over cosets of $\Gamma_{s-2}$ that are included in $\Gamma_{s-1}$. Writing each such difference of 
sums as a sum of differences gives the estimate

$$
\begin{aligned}
\left|\hat{f}_{s}\left(\chi_{j}\right)\right| & \leq\left(\frac{1}{m_{s-2}}\right)^{2}\left\{\sum_{0 \leq i<m_{s-2}}\left[\sum_{i \leq n<m_{s-1}}\left|\Delta a_{n}\right|\right]\right\} \\
& \leq\left(\frac{1}{m_{s-2}}\right)^{2}\left\{\sum_{n<m_{s-2}}(n+1)\left|\Delta a_{n}\right|+m_{s-2} \sum_{m_{s-2} \leq n<m_{s-1}}\left|\Delta a_{n}\right|\right\}
\end{aligned}
$$

for all $j$. Since $\hat{f}_{s}$ vanishes off $\Gamma_{s} \backslash \Gamma_{s-1}$ the $L^{2}$-norm of $f_{s}$ is at most $m_{s}^{1 / 2}$ times the quantity in the second line above, and the norm of $f_{s}$ in $B(1 / 2,2,1)$ is at most $m_{s}$ times the same quantity. Adding these estimates and interchanging the order of summation shows that if the function $a$ satisfies condition $F_{1}$ and the Vilenkin group is bounded then the series $\sum_{s=2}^{\infty} f_{s}$ converges in the space $B(1 / 2,2,1)$ to a function that satisfies condition (iii) in Theorem 2. An appeal to Theorem 3 now completes the proof of Theorem 4.

The only property of the function $a$ that was used in the analysis of the functions $f_{s}$ was that it satisfied condition $\mathcal{F}_{1}$. For later use we now summarize this and other consequences of condition $\mathcal{F}_{1}$. For each $r \geq 1$ let $h_{r}$ be the product $F_{r} \sum_{s=0}^{r-1} k_{s}$.

LEMMA 5. Let $G$ be a bounded Vilenkin group and let a be a function on $\Gamma$ that satisfies condition $\mathcal{F}_{1}$. Then the formal product $k \cdot F$ can be split into three parts in the following manner

(i) The series $\sum_{s=2}^{\infty} f_{s}$ converges in the space $B(1 / 2,2,1)$ to a function $f$ with the property that $S_{m_{t}} f(0)=0$ for all $t$.

(ii) The series $\sum_{s=2}^{\infty} k_{s} F_{s}$ converges in the space $B(1 / 2,2,1)$ to a function $g$ with the property that $\sum_{t=0}^{\infty}\left|S_{m_{t}} g(0)\right|<\infty$.

(iii) The series $\sum_{r=2}^{\infty} h_{r}$ converges in the space $B(0, \infty, 1)$ to a function $h$ with the property that $S_{m_{t}} h(0)=0$ for all $t$.

PROOF. Part (i) is just a summary of what we showed in the proof of Theorem 4. For part (ii) we begin with the observation that the transform of the product $k_{s} F_{s}$ vanishes off the subgroup $\Gamma_{s}$. On the other hand it follows from an analysis of expression (u) with $b=a$ on $\Gamma_{s} \backslash \Gamma_{s-1}$ and $b=0$ elsewhere that

$$
\left|\left[k_{s} F_{s}\right]^{\sim}\left(\chi_{j}\right)\right| \leq \frac{1}{m_{s-2}} \sum_{m_{s-1} \leq n<m_{s}}\left|\Delta a_{n}\right|
$$

for all $j<m_{s}$. The norm of any function in $B(1 / 2,2,1)$ is computed by finding the $l^{2}$-norm of the restriction of the transform of the function to each corona $\Gamma_{t} \backslash \Gamma_{t-1}$, multiplying this $l^{2}$-norm by $m_{t}^{1 / 2}$, and adding. Hence $\left\|k_{s} F_{s}\right\|_{B(1 / 2,2,1)}$ 
is majorized by the quantity on the right above multiplied by $\sum_{t=0}^{s} m_{t}$. Since the group is bounded

$$
\left\|k_{s} F_{s}\right\|_{B(1 / 2,2,1)} \leq c \sum_{m_{*}-1 \leq n<m_{s}}\left|\Delta a_{n}\right| .
$$

It follows that the series $\sum_{s=2}^{\infty} k_{s} F_{s}$ does indeed converge in the space $B(1 / 2,2,1)$.

Now consider the partial sums $S_{m_{t}}\left[k_{s} F_{s}\right](0)$. In the proof of Theorem 4 we showed that $S_{m_{s}}\left[k_{s} F_{r}\right](0)=0$ for all $r<s$. The same argument applies to $S_{m,}\left[k_{s} F_{s}\right](0)$ and since the transform of $k_{s} F_{s}$ vanishes outside the group $\Gamma_{s}$ we have that $S_{m_{t}}\left[k_{s} F_{s}\right](0)$ is 0 for all $t \geq s$. The corresponding partial sums with $t<s$ do not have to vanish at 0 but we can apply the estimate (9) above to get that

$$
\left|S_{m_{t}}\left[k_{s} F_{s}\right](0)\right| \leq c \frac{m_{t}}{m_{s}} \sum_{m_{s-1} \leq n<m_{s}}\left|\Delta a_{n}\right|
$$

for all $t<s$. It follows that

$$
\left|S_{m_{t}} g(0)\right| \leq c \sum_{s=t}^{\infty} \frac{m_{t}}{m_{s}} \sum_{m_{s-1} \leq n<m_{s}}\left|\Delta a_{n}\right|
$$

for each $t$. The desired conclusion that $\sum_{t=0}^{\infty}\left|S_{m_{\mathrm{t}}} g(0)\right|<\infty$ follows by adding with respect to $t$ and reversing the order of summation.

Finally we consider part (iii). When $s<r$ the transform of $k_{s} F_{r}$ vanishes off $\Gamma_{r} \backslash \Gamma_{r-1}$. This fact and an analysis of expression (7) again shows that the partial sums $S_{m_{t}}\left(k_{s} F_{r}\right)(0)$ are all zero. Hence the same is true with $k_{s} F_{r}$ replaced by $h_{r}$. Moreover $\hat{h}_{r}$ vanishes outside $\Gamma_{r} \backslash \Gamma_{r-1}$ so that $\left\|h_{r}\right\|_{B(0, \infty, 1)}=\left\|h_{r}\right\|_{\infty}$. Since the various functions $k_{S}$ have disjoint supports $\left\|h_{r}\right\|_{\infty}$ is the maximum of the quantities $\left\|k_{s} F_{r}\right\|_{\infty}$ for $s<r$. In expression (7) for such a product $k_{s} F_{r}$ the function $b$ coincides with $a$ on $\Gamma_{r} \backslash \Gamma_{r-1}$ and vanishes elsewhere, and the two cosets $\bar{\phi}_{s} \chi_{j} \Gamma_{s-2}$ and $\bar{\phi}_{s} \phi_{s-1} \chi_{j} \Gamma_{s-2}$ are both included in the same coset of $\Gamma_{s-1}$. It follows that

$$
\left|\left[k_{s} F_{r}\right]^{\mathcal{Y}}\left(\chi_{j}\right)\right| \leq \frac{1}{m_{s-2}} \sum_{\chi_{n} \in \bar{\phi}_{o} \chi_{j} \Gamma_{s-1}}\left|\Delta a_{n}\right|
$$

for all $j$ in $\left[m_{r-1}, m_{r}\right)$. Since $\left[k_{s} F_{r}\right]^{\wedge}\left(\chi_{j}\right)=0$ for all other values of $j$

$$
\left\|\left[k_{s} F_{r}\right]^{\sim}\right\|_{1} \leq \frac{m_{s-1}}{m_{s-2}} \sum_{m_{r-1} \leq n<m_{r}}\left|\Delta a_{n}\right| .
$$

Because the group is bounded and the norm $\left\|h_{r}\right\|_{B(0, \infty, 1)}$ is majorized by the maximum for $s<r$ of the lefthand sides above, the series $\sum_{r=2}^{\infty} h_{r}$ converges in the space $B(0, \infty, 1)$. The sum $h$ of the series will have the property that $S_{m_{t}} h(0)=0$ for all $t$. This completes the proof of the lemma.

To prove Theorem 1 split the formal product $k \cdot F$ into the pieces $f, g$ and $h$ specified by the lemma. By the argument used in the proof of Theorem 3 it 
sufficies to show that each of the functions $f / k, g / k$ and $h / k$ belongs to $L^{1}(G)$. That this is so for $f / k$ and $g / k$ follows from Theorem 3 and parts (i) and (ii) of the lemma. By part (iii) of the comment made at the end of the previous section all that remains is to verify that

$$
\sum_{r=1}^{\infty} \int_{G \backslash G_{r}}\left|h_{r}(x)\right| \frac{d x}{d(x, 0)}<\infty .
$$

To this end split the integral over $G \backslash G_{r}$ into integrals over coronas $G_{s-2} \backslash G_{s-1}$. On such a corona the only term in the sum for $h_{r}$ that is nonzero is $k_{s} F_{r}$. By Hölder's inequality

$$
\begin{aligned}
\int_{G_{s-2} \backslash G_{s-1}}\left|h_{r}(x)\right| \frac{d x}{d(x, 0)} & \leq\left\|k_{s} F_{r}\right\|_{p^{\prime}}\left\{\int_{G_{s-2} \backslash G_{s-1}} m_{s-1}^{p} d x\right\}^{1 / p} \\
& \leq c m_{s-1}^{1 / p^{\prime}}\left\|k_{s} F_{r}\right\|_{p^{\prime}} .
\end{aligned}
$$

Here $p^{\prime}$ denotes the index conjugate to $p$. Suppose without loss of generality that $p \in(1,2]$. Then the Hausdorff-Young theorem provides an estimate for the $L^{p^{\prime}}$-norm appearing above in terms of the $l^{p}$-norm of the Fourier coefficients of $k_{s} F_{r}$. Inequality $(10)$ shows that these coefficients can be individually estimated in terms of the $l^{1}$-norm of the restriction of the sequence $\left(\Delta a_{n}\right)$ to an appropriate coset of $\Gamma_{s-1}$. By Hölder's inequality again such an $l^{1}$-norm is majorized by $m_{s-1}^{1 / p^{\prime}}$ times the $l^{p}$-norm of the restriction of $\left(\Delta a_{n}\right)$ to the same coset. Combining these estimates yields that (the constant $c$ varies from line to line)

$$
\int_{G_{s-2} \backslash G_{s-1}}\left|h_{r}(x)\right| \frac{d x}{d(x, 0)} \leq c m_{s-1}^{1 / p^{\prime}}\left\{\sum_{m_{r-1} \leq n<m_{r}}\left|\Delta a_{n}\right|^{p}\right\}^{1 / p} .
$$

Adding as the index $s$ runs from 2 to $r+1$ gives

$$
\int_{G \backslash G_{r}}\left|h_{r}(x)\right| \frac{d x}{d(x, 0)} \leq c m_{r}^{1 / p^{\prime}}\left\{\sum_{m_{r-1} \leq n<m_{r}}\left|\Delta a_{n}\right|^{p}\right\}^{1 / p} .
$$

Finally adding with respect to $r$ yields inequality (11), thus completing the proof of Theorem 1.

Under condition $\mathcal{F}$ the coefficient sequence must satisfy $\mathcal{F}_{p}$ for some $p>1$, which is a stronger restriction than condition $\mathcal{F}_{1}$ alone. Integrability also follows from another condition that is stronger than $\mathcal{F}_{1}$.

THEOREM 6. If $\left(\chi_{n}\right)$ is a bounded Vilenkin system and if $\left(a_{n}\right)$ is a sequence of scalars satisfying the condition that

$$
\sum_{n=1}^{\infty}\left|\Delta a_{n}\right| \log n<\infty
$$

then the series $\sum_{n=0}^{\infty} a_{n} \chi_{n}$ represents an integrable function. 
Proof. To verify this note that in the proof of Theorem 1 only condition $\mathcal{F}_{1}$ was used except in the last step concerning inequality (11). Much as above each of the integrals $\int_{G \backslash G_{r}}\left|k_{s} F_{r}\right| d x / d(x, 0)$ with $s<r$ is majorized by $c \sum_{m_{r-1} \leq n<m_{r}}\left|\Delta a_{n}\right|$. Hence

$$
\int_{G \backslash G_{r}}\left|h_{r}(x)\right| \frac{d x}{d(x, 0)} \leq c(r-1) \sum_{m_{r-1} \leq n_{<m_{r}}}\left|\Delta a_{n}\right| .
$$

Inequality (11) now follows by adding with respect to $r$.

COROLLARY 7. If $\left(\chi_{n}\right)$ is a bounded Vilenkin system and if $\left(a_{n}\right)$ is a realvalued sequence that tends monotonically to 0 and satisfies the condition that

$$
\sum_{n=1}^{\infty} \frac{\left|a_{n}\right|}{n}<\infty
$$

then the series $\sum_{n=0}^{\infty} a_{n} \chi_{n}$ represents an integrable function.

PROOF. Summation by parts shows that the condition above and condition (12) are equivalent for real-valued sequences $\left(a_{n}\right)$ that tend monotonically to 0 .

We conclude the paper with some comments about other methods and results.

1. Our methods work with the function $k$ replaced by certain other adapted fucntions. The properties of $k$ that seem essential are that it be adapted, that the functions $k_{s}$ be supported on distinct coronas, and that the transforms $\hat{k}_{s}$ be supported on distinct coronas.

2. In every case that we have considered our results can also be proved by more direct methods, and in fact this has been done for Walsh series. As mentioned in the introduction it was already known [10] that a Walsh series with a sequence of coefficients that is quasiconvex and tends to 0 must be integrable, and Bruce Aubertin has a direct proof of Theorem 1 for Walsh series. Corollary 7 for Walsh series in the Kaczmarcz ordering was proved by Balashov [2]; his proof also works for the Paley ordering specified in Section 2.

3. We feel that our method of proof is of independent interest. Our proof of Theorem 1 could be shortened if we could show that the function $h$ must also belong to one of the spaces $B(1 / p, p, 1)$ with $p<\infty$. Unfortunately this conclusion about $h$ does not follow from the hypotheses of the theorem. There are convex sequences $\left(a_{n}\right)$ that tend to 0 for which the corresponding functions $h$ on the Walsh group belong to $B(1 / p, p, 1)$ only when $p=\infty$; such coefficient sequences satisfy condition $\mathcal{F}_{\infty}$ and hence $\mathcal{F}_{q}$ for all $q$. 
4. It is known [9] that the Dirichlet kernels $D_{N}$ on a Vilenkin group have the property that for each $x \neq 0$ the sequence $\left(D_{N}(x)\right)_{N=0}^{\infty}$ is bounded. It follows by summation by parts that if the sequence $\left(a_{n}\right)$ tends to 0 and satisfies condition $f_{1}$ then the series $\sum_{n=0}^{\infty} a_{n} \chi_{n}(x)$ converges pointwise except at $x=0$. Our theorems provide criteria for this pointwise sum to belong to $L^{1}(G)$.

5. Our methods also lead to estimates for the $L^{1}$-norm of the function represented by the series. Under the hypotheses of Theorem 1 we get that

$$
\|f\|_{1} \leq c_{p}(G) \sum_{r=1}^{\infty} m_{r}\left\{\frac{1}{m_{r}} \sum_{m_{r-1} \leq n<m_{r}}\left|\Delta a_{n}\right|^{p}\right\}^{1 / p}
$$

for all $p>1$. Similarly Corollary 7 can be recast to assert that

$$
\|f\|_{1} \leq c(G)\left[\left|a_{0}\right|+\sum_{n=1}^{\infty} \frac{\left|a_{n}\right|}{n}\right]
$$

whenever the right hand side is finite and the sequence $\left(a_{n}\right)$ is real-valued and tends monotonically to 0 . It follows from inequality (13) that the Fejér kernels $K_{N}$ on a bounded Vilenkin group $G$ form a bounded sequence in the space $L^{1}(G)$; this conclusion is implicit in [9]. Applying inequality (14) to the Dirichlet kernels on a bounded Vilenkin group yields that $\left\|D_{N}\right\|_{1} \leq c(G) \log N$ for all $N$.

6. This conclusion about the Dirichlet kernels was shown by Vilenkin [9] to hold without the requirement that the group be bounded. The conclusion about the $l^{1}$-norms of the Fejér kernels also holds for unbounded groups. We have used boundedness repeatedly in this paper, and we do not know whether our other conclusions continue to hold without this assumption.

\section{References}

[1] A. S. Baiarstanova, 'On a class of series with monotone coefficients', Izv. Vysh. Uchebn. Zaved. Mat. 27 (12) (1983), 3-7; translated in Soviet Math. (Izv. VUZ) 27 (12) (1983), 3-7.

[2] L. A. Balashov, 'On series with respect to the Walsh system with monotone coefficients', Sibirsk. Mat. Zh. 12 (1971), 25-39; translated in Siberian Math. J. 12 (1971), 18-28.

[3] Walter R. Bloom and John J. F. Fournier, 'Generalized Lipschitz spaces on Vilenkin groups', Math. Nachr. 132 (1987), 67-80.

[4] William O. Bray and Vera B. Stanojević, 'On the integrability of complex trigonometric series', Proc. Amer. Math. Soc. 93 (1985), 51-58.

[5] R. E. Edwards, Fourier series, a modern introduction (Holt, Rinehart and Winston, New York, 1967).

[6] G. A. Fomin, 'A class of trigonometric series', Mat. Zametki 23 (1978), 213-222; translated in Math. Notes 23 (1978), 117-123.

[7] John J. F. Fournier and W. Self, 'Some sufficient conditions for uniform convergence of Fourier series', J. Math. Anal. Appl. 126 (1987), 355-374. 
[8] C. W. Onneweer, 'On the definition of dyadic differantiation', Applicable Anal. 9 (1979), 267-278.

[9] N. Ya. Vilenkin, 'On a class of complete orthonormal systems', Izv. Akad. Nauk SSSR Ser. Mat. 11 (1947), 363-400; translated in Amer. Math. Soc. Transl. 28 (1963), 1-35.

[10] Shigeki Yano, 'On Walsh-Fourier series', Tôhoku Math. J. 3 (1951), 223-242.

School of Mathematical

and Physical Sciences

Murdoch University

Perth, Western Australia 6150

Australia
Department of Mathematics

University of British Columbia

Vancouver, Canada

V6T 1Y4 